\title{
Members of random closed sets
}

\author{
David Diamondstone $^{1}$ and Bjørn Kjos-Hanssen ${ }^{2}$ \\ 1 Department of Mathematics, University of Chicago, Chicago IL 60615 \\ ded@math.uchicago.edu \\ http://www.math.uchicago.edu/ ded \\ 2 Department of Mathematics, University of Hawai'i at Mānoa, Honolulu HI 96822 \\ bjoern@math.hawaii.edu \\ http://www.math.hawaii.edu/ bjoern
}

\begin{abstract}
The members of Martin-Löf random closed sets under a distribution studied by Barmpalias et al. are exactly the infinite paths through Martin-Löf random Galton-Watson trees with survival parameter $\frac{2}{3}$. To be such a member, a sufficient condition is to have effective Hausdorff dimension strictly greater than $\gamma=\log _{2} \frac{3}{2}$, and a necessary condition is to have effective Hausdorff dimension greater than or equal to $\gamma$.

Keywords: random closed sets, computability theory.
\end{abstract}

\section{Introduction}

Classical probability theory studies intersection probabilities for random sets. A random set will intersect a given deterministic set if the given set is large, in some sense. Here we study a computable analogue: the question of which real numbers are "large" in the sense that they belong to some Martin-Löf random closed set.

Barmpalias et al. 2 introduced algorithmic randomness for closed sets. Subsequently Kjos-Hanssen [6] used algorithmically random Galton-Watson trees to obtain results on infinite subsets of random sets of integers. Here we show that the distributions studied by Barmpalias et al. and by Galton and Watson are actually equivalent, not just classically but in an effective sense.

For $0 \leq \gamma<1$, let us say that a real $x$ is a MembeR $\gamma$ if $x$ belongs to some Martin-Löf (ML-) random closed set according to the Galton-Watson distribution (defined below) with survival parameter $p=2^{-\gamma}$. We show that for $p=\frac{2}{3}$, this is equivalent to $x$ being a member of a Martin-Löf random closed set according to the distribution considered by Barmpalias et al.

In light of this equivalence, we may state that (i) Barmpalias et al. showed

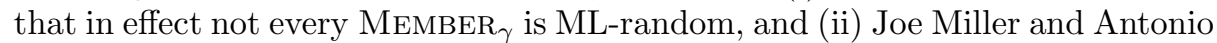
Montálban showed that every ML-random real is a MEMBER $\gamma$; the proof of their result is given in the paper of Barmpalias et al. 22 The way to sharpen these results go via effective Hausdorff dimension. Each ML-random real has effective Hausdorff dimension equal to one. In Section 3 we show that $\left(i^{\prime}\right)$ a MEMBER $\gamma$ may have effective Hausdorff dimension strictly less than one, and (ii') every real 
of sufficiently large effective Hausdorff dimension (where some numbers strictly less than one are "sufficiently large") is a MEMBER $\gamma$.

\section{Equivalence of two models}

We write $\Omega=2^{<\omega}$, and $2^{\omega}$, for the sets of finite and infinite strings over $2=$ $\{0,1\}$, respectively. If $\sigma \in \Omega$ is an initial substring (a prefix) of $\tau \in \Omega$ we write $\sigma \preceq \tau$; similarly $\sigma \prec x$ means that the finite string $\sigma$ is a prefix of the infinite string $x \in 2^{\omega}$. The length of $\sigma$ is $|\sigma|$. We use the standard notation $[\sigma]=\{x: \sigma \prec x\}$, and for a set $U \subseteq \Omega,[U] \preceq:=\bigcup_{\sigma \in U}[\sigma]$. Let $\mathcal{P}$ denote the power set operation. Following Kjos-Hanssen [6], for a real number $0 \leq \gamma<1$ (so $\frac{1}{2}<2^{-\gamma} \leq 1$ ), let $\lambda_{1, \gamma}$ be the distribution with sample space $\mathcal{P}(\Omega)$ such that each string in $\Omega$ has probability $2^{-\gamma}$ of belonging to the random set, independently of any other string. Let $\lambda_{\gamma}^{*}$ be defined analogously, except that now

$$
\lambda_{\gamma}^{*}\left(\{S: S \cap\{\sigma 0, \sigma 1\}=J\}= \begin{cases}1-p & \text { if } J=\{\sigma 0\} \text { or } J=\{\sigma 1\}, \text { and } \\ 2 p-1 & \text { if } J=\{\sigma 0, \sigma 1\},\end{cases}\right.
$$

independently for distinct $\sigma$, for $p=2^{-\gamma}$. 11 For $S \subseteq \Omega, \Gamma_{S}$, the closed set determined by $S$, is the (possibly empty) set of infinite paths through the part of $S$ that is downward closed under prefixes:

$$
\Gamma_{S}=\left\{x \in 2^{\omega}:(\forall \sigma \prec x) \sigma \in S\right\} .
$$

The Galton-Watson ( $G W$ ) distribution for survival parameter $2^{-\gamma}$, also known as the $(1, \gamma)$-induced distribution $[6$, and as the distribution of a percolation limit set $\left[12\right.$, is a distribution $\mathbb{P}_{1, \gamma}$ on the set of all closed subsets of $2^{\omega}$ defined by

$$
\mathbb{P}_{1, \gamma}(E)=\lambda_{1, \gamma}\left\{S: \Gamma_{S} \in E\right\} .
$$

Thus, the probability of a property $E$ of a closed subset of $2^{\omega}$ is the probability according to $\lambda_{1, \gamma}$ that a random subset of $\Omega$ determines a tree whose set of infinite paths has property $E$. Similarly, let

$$
\mathbb{P}_{\gamma}^{*}(E)=\lambda_{\gamma}^{*}\left\{S: \Gamma_{S} \in E\right\} .
$$

A $\Sigma_{1}^{0}$ subset of $\mathcal{P}(\Omega)$ is the image of a $\Sigma_{1}^{0}$ subset of $\mathcal{P}(\omega)=2^{\omega}$ via an effective isomorphism between $\Omega$ and $\omega$.

$S \in \mathcal{P}(\Omega)$ is called $\lambda_{1, \gamma}$-ML-random if for each uniformly $\Sigma_{1}^{0}$ sequence $\left\{U_{n}\right\}_{n \in \omega}$ of subsets of $\mathcal{P}(\Omega)$ with $\lambda_{1, \gamma}\left(U_{n}\right) \leq 2^{-n}$, we have $S \notin \bigcap_{n} U_{n}$. In this case $\Gamma_{S}$ is called $\mathbb{P}_{1, \gamma}$-ML-random. Similarly, $S \in \mathcal{P}(\Omega)$ is called $\lambda_{\gamma}^{*}$-MLrandom if for each uniformly $\Sigma_{1}^{0}$ sequence $\left\{U_{n}\right\}_{n \in \omega}$ of subsets of $\mathcal{P}(\Omega)$ with $\lambda_{\gamma}^{*}\left(U_{n}\right) \leq 2^{-n}$, we have $S \notin \bigcap_{n} U_{n}$. In this case $\Gamma_{S}$ is called $\mathbb{P}_{\gamma}^{*}$-ML-random.

\footnotetext{
${ }^{1}$ The notation $\lambda_{1, \gamma}$ is consistent with earlier usage 6] and is also easy to distinguish visually from $\lambda_{\gamma}^{*}$.
} 
Lemma 1 (Axon 1]). For $2^{-\gamma}=\frac{2}{3}, \Gamma \subseteq 2^{\omega}$ is $\mathbb{P}_{\gamma}^{*}-M L$-random if and only if $\Gamma$ is a Martin-Löf random closed set under the distribution studied by Barmpalias et al.

Thinking of $S$ as a random variable, define further random variables

$$
G_{n}=\{\sigma:|\sigma|=n \quad \& \quad(\forall \tau \preceq \sigma) \tau \in S\}
$$

and $G=\bigcup_{n=0}^{\infty} G_{n}$. We refer to a value of $G$ as a $G W$-tree when $G$ is considered a value of the random variable under the $\lambda_{1, \gamma}$ distribution. (A $B B C D W$-tree is a particular value of the random variable analogous to $G$, for the distribution $\lambda_{\gamma}^{*}$.) We have $G \subseteq S$ and $\Gamma_{G}=\Gamma_{S}$. The set $G$ may have "dead ends", so let

$$
G_{\infty}=\{\sigma \in G: G \cap[\sigma] \text { is infinite }\} .
$$

Thus $G_{\infty} \subseteq G \subseteq S$, and values of $G_{\infty}$ are in one-to-one correspondence with values of $\Gamma_{S}$.

Let $e$ be the extinction probability of a GW-tree with parameter $p=2^{-\gamma}$,

$$
e=\mathbb{P}_{1, \gamma}(\varnothing)=\lambda_{1, \gamma}\left(\left\{S: \Gamma_{S}=\varnothing\right\}\right) .
$$

For any number $a$ let $\bar{a}=1-a$.

\section{Lemma 2.}

$$
e=\bar{p} / p \text {. }
$$

Proof. Notice that we are not assuming \langle\rangle$\in S$. We have $e=\bar{p}+p e^{2}$, because there are two ways extinction can happen: $(1)\langle\rangle \notin S$, and $(2)\langle\rangle \in S$ but both immediate extension trees go extinct.

We use standard notation for conditional probability,

$$
\mathbb{P}(E \mid F)=\frac{\mathbb{P}(E \cap F)}{\mathbb{P}(F)} ;
$$

in measure notation we may also write $\lambda(E \mid F)=\lambda(E \cap F) / \lambda(F)$.

Lemma 3. For all $J \subseteq\{\langle 0\rangle,\langle 1\rangle\}$,

$$
\lambda_{1, \gamma}\left\{G_{\infty} \cap\{\langle 0\rangle,\langle 1\rangle\}=J \mid G_{\infty} \neq \varnothing\right\}=\lambda_{\gamma}^{*}\left[G_{1}=J\right] .
$$

Proof. By definition, $\lambda_{\gamma}^{*}\left[G_{1}=J\right]$ equals

$$
(2 p-1) \cdot \mathbf{1}_{J=\{\langle 0\rangle,\langle 1\rangle\}}+\sum_{i=0}^{1}(1-p) \cdot \mathbf{1}_{J=\{\langle i\rangle\}},
$$

so we only need to calculate $\lambda_{1, \gamma}\left\{G_{\infty} \cap\{\langle 0\rangle,\langle 1\rangle\}=J \mid G_{\infty} \neq \varnothing\right\}$. By symmetry, and because the probability that $G_{1}=\varnothing$ is 0 , it suffices to calculate this probability for $J=\{\langle 0\rangle,\langle 1\rangle\}$. Now if $G_{1}=\{\langle 0\rangle,\langle 1\rangle\}$ then \langle\rangle survives and both immediate extensions are non-extinct. Thus the conditional probability that $G_{1}=\{\langle 0\rangle,\langle 1\rangle\}$ is $\frac{p(1-e)^{2}}{1-e}=p(1-e)$. By Lemma 2 , this is equal to $2 p-1$. 
Lemma 4. Let the number $\mathfrak{p}_{s}$ be defined by

$$
\mathfrak{p}_{s}=\lambda_{1, \gamma}\left(\langle j\rangle \in G \mid(G \cap(\langle j\rangle \frown \Omega))_{\infty}=\varnothing \&\langle\rangle \in G\right)
$$

for $j=0$ (or $j=1$, which gives the same result). Let

$$
\lambda_{f}(\cdot)=\lambda_{1, \gamma}\left(\cdot \mid G_{\infty}=\varnothing \quad \&\langle\rangle \in G\right) .
$$

Then $\lambda_{f}\left(\langle i\rangle \in G_{1}\right)=\mathfrak{p}_{s}$.

Proof. We have $\mathfrak{p}_{s}=p^{2} e^{2} /(p e)=p e=1-p$. Next, $\lambda_{f}\left[G_{1}=\varnothing\right]=\frac{p(1-p)^{2}}{p e^{2}}=p^{2}$ and $\lambda_{f}\left[G_{1}=\{\langle 0\rangle,\langle 1\rangle\}\right]=\frac{p^{3} e^{4}}{p e^{2}}=(\bar{p})^{2}$. Hence

$$
\lambda_{f}\left[G_{1}=J\right]=(1-p)^{2} \cdot \mathbf{1}_{J=\{\langle 0\rangle,\langle 1\rangle\}}+\sum_{i=0}^{1} p(1-p) \cdot \mathbf{1}_{J=\{\langle i\rangle\}}+p^{2} \cdot \mathbf{1}_{J=\varnothing},
$$

and so $\lambda_{f}\left(\langle i\rangle \in G_{1}\right)=(1-p)^{2}+p(1-p)=\mathfrak{p}_{s}$, as desired.

Let $\lambda_{c}=\lambda_{1, \gamma}\left(\cdot \mid G_{\infty} \neq \varnothing\right)$ be $\lambda_{1, \gamma}$ conditioned on $G_{\infty} \neq \varnothing$, and let $\lambda_{i}$ be the distribution of $G_{\infty} \in \mathcal{P}(\Omega)$ conditional on $G_{\infty} \neq \varnothing$. Let $\mu_{i}, \mu_{f}, \mu_{c}$ be the distribution of the tree $G$ corresponding to the set $S$ under $\lambda_{i}, \lambda_{f}, \lambda_{c}$, respectively (so $\mu_{i}=\lambda_{i}$ ). We define a $\mu_{i} \times \mu_{f} \rightarrow \mu_{c}$ measure-preserving map $\psi: 2^{\Omega} \times 2^{\Omega} \rightarrow 2^{\Omega}$. The idea is to overlay two sets $S_{i}, S_{f}$, so that $S_{i}$ specifies $G_{\infty}$, and $S_{f}$ specifies $G \backslash G_{\infty}$. Let $\psi\left(S_{i}, S_{f}\right)=G_{i} \cup S_{f}$ where $G_{i}$ is the tree determined by $S_{i}$. By Lemma 4 , this gives the correct probability for string $\sigma \notin G_{\infty}$ that is the neighbor of a string in $G_{\infty}$ to be in $G$. By considering two cases (a string in $G$ is in $G_{\infty}$ or not) we can derive that $\psi$ is measure-preserving.

Intuitively, a $\lambda_{i}$-ML-random tree may by van Lambalgen's theorem be extended to a $\lambda_{c}$-ML-random tree by "adding finite pieces randomly". To be precise, van Lambalgen's theorem holds in the unit interval $[0,1]$ with Lebesgue measure $\lambda$, or equivalently the space $2^{\omega}$. If $(X, \mu)$ is a measure space then using the measure-preserving map $\varphi:(X, \mu) \rightarrow([0,1], \lambda)$ induced from the Carathéodory measure algebra isomorphism theorem [7, we may apply van Lambalgen as desired, and obtain

Theorem 1. For each $M L$-random $B B C D W$-tree $H$ there is a $M L$-random $G W$ tree $G$ with $G_{\infty}=H_{\infty}$.

We next prove that the live part of every infinite ML-random GW-tree is an ML-random BBCDW-tree.

Theorem 2. For each $S$, if $S$ is $\lambda_{1, \gamma}-M L$-random then $G_{\infty}$ is $\lambda_{\gamma}^{*}$-random.

Proof. Suppose $\left\{U_{n}\right\}_{n \in \omega}$ is a $\lambda_{\gamma}^{*}$-ML-test with $G_{\infty} \in \bigcap_{n} U_{n}$. Let $\Upsilon_{n}=\{S$ : $\left.G_{\infty} \in U_{n}\right\}$. By Lemma 3, $\lambda_{1, \gamma}\left(\Upsilon_{n}\right)=\lambda_{\gamma}^{*}\left(U_{n}\right)$. Unfortunately, $\Upsilon_{n}$ is not a $\Sigma_{1}^{0}$ class, but we can approximate it. While we cannot know if a tree will end up being infinite, we can make a guess that will usually be correct. 
Let $e$ be the probability of extinction for a GW-tree. By Lemma 2 we have $e=\frac{\bar{p}}{p}$, so since $p>1 / 2, e<1$. Thus there is a computable function $(n, \ell) \mapsto m_{n, \ell}$ such that for all $n$ and $\ell, m=m_{n, \ell}$ is so large that $e^{m} \leq 2^{-n} 2^{-2 \ell}$. Let $\Phi$ be a Turing reduction so that $\Phi^{G}(n, \ell)$, if defined, is the least $L$ such that all the $2^{\ell}$ strings of length $\ell$ either are not on $G$, or have no descendants on $G$ at level $L$, or have at least $m_{n, \ell}$ many such descendants. Let

$$
W_{n}=\left\{S: \text { for some } \ell, \Phi^{G}(n, \ell) \text { is undefined }\right\} .
$$

Let $A_{G}(\ell)=G_{\infty} \cap\{0,1\} \leq \ell$ be $G_{\infty}$ up to level $\ell$. Let the approximation $A_{G}(\ell, L)$ to $A_{G}(\ell)$ consist of the nodes of $G$ at level $\ell$ that have descendants at level $L$. Let

$$
V_{n}=\left\{S: A_{G}(\ell, L) \in U_{n} \text { for some } \ell, \text { where } L=\Phi^{G}(n, \ell)\right\}, \text { and }
$$

$X_{n}=\left\{S:\right.$ for some $\ell, L=\Phi^{G}(n, \ell)$ is defined and $\left.A_{G}(\ell, L) \neq A_{G}(\ell)\right\}$.

Note that $\Upsilon_{n}=\left\{S\right.$ : for some $\left.\ell, A_{G}(\ell) \in U_{n}\right\}$, hence $\Upsilon_{n} \subseteq W_{n} \cup X_{n} \cup V_{n}$. Thus it suffices to show that $\cap_{n} V_{n}, W_{n}, \cap_{n} X_{n}$ are all $\lambda_{1, \gamma}$-ML-null sets.

Lemma 5. $\lambda_{1, \gamma}\left(W_{n}\right)=0$.

Proof. If $\Phi(\ell)$ is undefined then there is no $L$, which means that for the fixed set of strings on $G$ at level $\ell$, they do not all either die out or reach $m$ many extensions. But eventually this must happen, so $L$ must exist.

Indeed, fix any string $\sigma$ on $G$ at level $\ell$. Let $k$ be the largest number of descendants that $\sigma$ has at infinitely many levels $L>\ell$. If $k>0$ then with probability 1 , above each level there is another level where actually $k+1$ many descendants are achieved. So we conclude that either $k=0$ or $k$ does not exist.

From basic computability theory, $W_{n}$ is a $\Sigma_{2}^{0}$ class. Hence each $W_{n}$ is a Martin-Löf null set.

Lemma 6. $\lambda_{1, \gamma}\left(X_{n}\right) \leq 2^{-n}$.

Proof. Let $E_{\sigma}$ denote the event that all extensions of $\sigma$ on level $L$ are dead, i.e. not in $G_{\infty}$. Let $F_{\sigma}$ denote the event that $\sigma$ has at least $m$ many descendants on $G(L)$.

If $A_{G}(\ell, L) \neq A_{G}(\ell)$ then some $\sigma \in\{0,1\}^{\ell} \cap G$ has at least $m$ many descendants at level $L$, all of which are dead. If a node $\sigma$ has at least $m$ descendants, then the chance that all of these are dead, given that they are on $G$ at level $L$, is at most $e^{m}$ (the eventual extinction of one is independent of that of another), hence writing $\mathbb{P}=\lambda_{1, \gamma}$, we have

$$
\begin{gathered}
\mathbb{P}\left(A_{G}(\ell, L) \neq A_{G}(\ell)\right) \leq \sum_{\sigma \in\{0,1\}^{\ell}} \mathbb{P}\left\{E_{\sigma} \quad \& F_{\sigma}\right\}=\sum_{\sigma \in\{0,1\}^{\ell}} \mathbb{P}\left\{E_{\sigma} \mid F_{\sigma}\right\} \cdot \mathbb{P}\left\{F_{\sigma}\right\} \\
\leq \sum_{\sigma \in\{0,1\}^{\ell}} \mathbb{P}\left\{E_{\sigma} \mid F_{\sigma}\right\} \leq \sum_{\sigma \in\{0,1\}^{\ell}} e^{m} \leq \sum_{\sigma \in\{0,1\}^{\ell}} 2^{-n} 2^{-2 \ell}=2^{-n} 2^{-\ell}
\end{gathered}
$$


and hence

$$
\mathbb{P} X_{n} \leq \sum_{\ell} \mathbb{P}\left\{A_{G}(\ell, L) \neq A_{G}(\ell)\right\} \leq \sum_{\ell} 2^{-n} 2^{-\ell}=2^{-n} .
$$

$X_{n}$ is $\Sigma_{1}^{0}$ since when $L$ is defined, $A_{G}(\ell)$ is contained in $A_{G}(\ell, L)$, and $A_{G}(\ell)$ is $\Pi_{1}^{0}$ in $G$, which means that if the containment is proper then we can eventually enumerate (observe) this fact. Thus $\cap_{n} X_{n}$ is a $\lambda_{1, \gamma}$-ML-null set.

$V_{n}$ is clearly $\Sigma_{1}^{0}$. Moreover $V_{n} \subseteq \Upsilon_{n} \cup X_{n}$, so $\lambda_{1, \gamma}\left(V_{n}\right) \leq 2 \cdot 2^{-n}$, hence $\cap_{n} V_{n}$ is a $\lambda_{1, \gamma}$-ML-null set.

\section{Towards a characterization of members of random closed sets}

For a real number $0 \leq \gamma \leq 1$, the $\gamma$-weight $\mathrm{wt}_{\gamma}(C)$ of a set of strings $C \subseteq \Omega$ is defined by

$$
\mathrm{wt}_{\gamma}(C)=\sum_{w \in C} 2^{-|w| \gamma} .
$$

We define several notions of randomness of individual reals. A Martin-Löf (ML-) $\gamma$-test is a uniformly $\Sigma_{1}^{0}$ sequence $\left(U_{n}\right)_{n<\omega}, U_{n} \subseteq \Omega$, such that for all $n$, $\mathrm{wt}_{\gamma}\left(U_{n}\right) \leq 2^{-n}$. A strong $M L-\gamma$-test is a uniformly $\Sigma_{1}^{0}$ sequence $\left(U_{n}\right)_{n<\omega}$ such that for each $n$ and each prefix-free set of strings $V_{n} \subseteq U_{n}, \mathrm{wt}_{\gamma}\left(V_{n}\right) \leq 2^{-n}$. A real is (strongly) $\gamma$-random if it does not belong to $\cap_{n}\left[U_{n}\right] \preceq$ for any (strong) ML- $\gamma$-test $\left(U_{n}\right)_{n<\omega}$. If $\gamma=1$ we simply say that the real, or the set of integers $\{n: x(n)=1\}$, is Martin-Löf random (ML-random). For $\gamma=1$, strength makes no difference. For a measure $\mu$ and a real $x$, we say that $x$ is Hippocrates $\mu$-random if for each sequence $\left(U_{n}\right)_{n<\omega}$ that is uniformly $\Sigma_{1}^{0}$, and where $\mu\left[U_{n}\right] \preceq \leq 2^{-n}$ for all $n$, we have $x \notin \cap_{n}\left[U_{n}\right]^{\preceq}$. Let the ultrametric $v$ on $2^{\omega}$ be defined by $v(x, y)=2^{-\min \{n: x(n) \neq y(n)\}}$. The $\gamma$-energy 12 of a measure $\mu$ is

$$
I_{\gamma}(\mu):=\iint \frac{d \mu(b) d \mu(a)}{v(a, b)^{\gamma}} .
$$

$x$ is Hippocrates $\gamma$-energy random if $x$ is Hippocrates $\mu$-random with respect to some probability measure $\mu$ such that $I_{\gamma}(\mu)<\infty$.

For background on $\gamma$-energy and related concepts the reader may consult the monographs of Falconer [3] and Mattila [11] or the on-line lecture notes of Mörters and Peres [12. The terminology Hippocrates random is supposed to remind us of Hippocrates, who did not consult the oracle at Delphi, but instead looked for natural causes. An almost sure property is more effective if it is possessed by all Hippocrates $\mu$-random reals rather than merely all $\mu$ random reals. In this sense Hippocratic $\mu$-randomness tests are more desirable than arbitrary $\mu$-randomness tests.

Effective Hausdorff dimension was introduced by Lutz 8 and is a notion of partial randomness. For example, if the sequence $x_{0} x_{1} x_{2} \cdots$ is ML-random, then the sequence $x_{0} 0 x_{1} 0 x_{2} 0 \cdots$ has effective Hausdorff dimension equal to $\frac{1}{2}$. 
Let $\operatorname{dim}_{H}^{1} x$ denote the effective (or constructive) Hausdorff dimension of $x$; then we have $\operatorname{dim}_{H}^{1}(x)=\sup \{\gamma: x$ is $\gamma$-random $\}$ (Reimann and Stephan 14]).

Examples of measures of finite $\gamma$-energy may be obtained from the fact that if $\operatorname{dim}_{H}^{1}(x)>\gamma$ then $x$ is Hippocrates $\gamma$-energy random 66. If $x$ is strongly

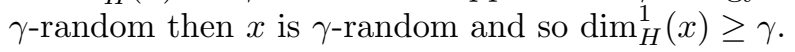

Theorem 3 ([6]). Each Hippocrates $\gamma$-energy random real is a $\mathrm{MEMBER}_{\gamma}$.

Here we show a partial converse:

Theorem 4. Each $\mathrm{MEMBER}_{\gamma}$ is strongly $\gamma$-random.

Proof. Let $\mathbb{P}=\lambda_{1, \gamma}$ and $p=2^{-\gamma} \in\left(\frac{1}{2}, 1\right]$. Let $i<2$ and $\sigma \in \Omega$. The probability that the concatenation $\sigma i \in G$ given that $\sigma \in G$ is by definition

$$
\mathbb{P}\{\sigma i \in G \mid \sigma \in G\}=p .
$$

Hence the absolute probability that $\sigma$ survives is

$$
\mathbb{P}\{\sigma \in G\}=p^{|\sigma|}=\left(2^{-\gamma}\right)^{|\sigma|}=\left(2^{-|\sigma|}\right)^{\gamma} .
$$

Let $U$ be any strong $\gamma$-test, i.e. a uniformly $\Sigma_{1}^{0}$ sequence $U_{n}=\left\{\sigma_{n, i}: i<\omega\right\}$, such that for all prefix-free subsets $U_{n}^{\prime}=\left\{\sigma_{n, i}^{\prime}: i<\omega\right\}$ of $U_{n}$, wt ${ }_{\gamma}\left(U_{n}^{\prime}\right) \leq 2^{-n}$. Let $U_{n}^{\prime}$ be the set of all strings $\sigma$ in $U_{n}$ such that no prefix of $\sigma$ is in $U_{n}$. Clearly, $U_{n}^{\prime}$ is prefix-free. Let

$$
\left[V_{n}\right]^{\preceq}:=\left\{S: \exists i \sigma_{n, i} \in G\right\} \subseteq\left\{S: \exists i \sigma_{n, i}^{\prime} \in G\right\} .
$$

Clearly $\left[V_{n}\right] \preceq$ is uniformly $\Sigma_{1}^{0}$. To prove the inclusion: Suppose $G$ contains some $\sigma_{n, i}$. Since $G$ is a tree, it contains the shortest prefix of $\sigma_{n, i}$ that is in $U_{n}$, and this string is in $U_{n}^{\prime}$. Now

$$
\mathbb{P}\left[V_{n}\right]^{\preceq} \leq \sum_{i \in \omega} \mathbb{P}\left\{\sigma_{n, i}^{\prime} \in G\right\}=\sum_{i \in \omega} 2^{-\left|\sigma_{n, i}^{\prime}\right| \gamma} \leq 2^{-n} .
$$

Thus $V$ is a test for $\lambda_{1, \gamma}$-ML-randomness. Suppose $x$ is a Member $\gamma$. Let $S$ be any $\lambda_{1, \gamma}$-ML-random set with $x \in \Gamma_{S}$. Then $S \notin \cap_{n}\left[V_{n}\right] \preceq$, and so for some $n$, $\Gamma \cap\left[U_{n}\right]^{\preceq}=\varnothing$. Hence $x \notin\left[U_{n}\right]^{\preceq}$. As $U$ was an arbitrary strong $\gamma$-test, this shows that $x$ is strongly $\gamma$-random.

Corollary 1. Let $x \in 2^{\omega}$. We have the implications

$$
\operatorname{dim}_{H}^{1}(x)>\gamma \Rightarrow x \text { is a } \operatorname{MEMBER}_{\gamma} \Rightarrow \operatorname{dim}_{H}^{1}(x) \geq \gamma .
$$

Proof. Each real $x$ with $\operatorname{dim}_{H}^{1}(x)>\gamma$ is $\beta$-capacitable for some $\beta>\gamma$ 13. This implies that $x$ is $\gamma$-energy random [6, Lemma 2.5] and in particular $x$ is Hippocrates $\gamma$-energy random. This gives the first implication. For the second implication, we use the fact that each strongly $\gamma$-random real $x$ satisfies $\operatorname{dim}_{H}^{1}(x) \geq \gamma($ see e.g. Reimann and Stephan 14 ). 
The second implication of Corollary 1 does not reverse, as not every real with $\operatorname{dim}_{H}^{1}(x) \geq \gamma$ is strongly $\gamma$-random 14 .

The first implication of Corollary 1 fails to reverse as well:

Proposition 1. Let $0<\gamma<1$. There is a $\gamma$-energy random real of effective Hausdorff dimension exactly $\gamma$.

Proof. Consider the probability measure $\mu$ on $2^{\omega}$ such that $\mu\left(\left[\sigma^{\frown} 0\right]\right)=\mu\left(\left[\sigma^{\frown} 1\right]\right)$ for all $\sigma$ of even length, and such that $\mu([\sigma \frown 0])=\mu([\sigma])$ for each $\sigma$ of odd length $f(k)=2 k+1$. A computation shows that $I_{\gamma}(\mu)=\sum_{k=0}^{\infty} 2^{(2 k+1) \gamma} 2^{-k}$ which is finite if and only if $\gamma<1 / 2$. We find that $\mu$-almost all reals are $\mu$ random and have effective Hausdorff dimension exactly $1 / 2$. By modifying $f(k)$ slightly we can get $I_{\gamma}(\mu)<\infty$ for $\gamma=1 / 2$ while keeping the effective Hausdorff dimension of $\mu$-almost all reals equal to $1 / 2$. Namely, what is needed is that $\sum_{k=0}^{\infty} 2^{f(k) \gamma} 2^{-k}<\infty$. This holds if $\gamma=1 / 2$ and $f(k)=2 k-2(1-\varepsilon) \log k$ for any $\varepsilon>0$ since $\sum_{k} k^{-(1+\varepsilon)}<\infty$. Since this $f(k)$ is asymptotically larger than $(2-\delta) k$ for any $\delta>0$, the $\mu$-random reals still have effective Hausdorff dimension $1 / 2$. The example generalizes from $\gamma=1 / 2$ to an arbitrary $0<\gamma<1$. have

Writing implication known to be strict as $\Rightarrow$ and other implication as $\rightarrow$, we

$\operatorname{dim}_{H}^{1}(x)>\gamma \Rightarrow x$ is $\gamma$-energy random $\rightarrow x$ is Hippocrates $\gamma$-energy

random $\rightarrow x$ is a MEMBER $\gamma \rightarrow x$ is strongly $\gamma$-random $\Rightarrow x$ is $\gamma$-random

$\Rightarrow \operatorname{dim}(x) \geq \gamma$.

By Reimann's effective capacitability theorem $[13 x$ is strongly $\gamma$-random if and only if $x$ is $\gamma$-capacitable.

Conjecture 1. There is a strongly $\gamma$-random real which is not Hippocrates $\gamma$ energy random.

Conjecture 2. A real $x$ is a MEMBER $_{\gamma}$ if and only if $x$ is Hippocrates $\gamma$-energy random.

To prove Conjecture 2, one might try to consult Lyons [10].

Proposition 2. Let $0<\gamma<1$. If $x$ is a real such that the function $n \mapsto$ $x(n)$ is f-computably enumerable for some computable function $f$ for which $\sum_{j<n} f(i) 2^{-n \gamma}$ goes effectively to zero, then $x$ is not $\gamma$-random.

Proof. Suppose $n \mapsto x(n)$ is $f$-c.e. for some such $f$, and let $F(n)=\sum_{j<n} f(n)$. Let $\alpha$ be any computable function such that $\alpha(n, i) \neq \alpha(n, i+1)$ for at most $f(n)$ many $i$ for each $n$, and $\lim _{i \rightarrow \infty} \alpha(i, n)=x(n)$. Let $c(n, j)$ be the $j$ th such $i$ that is discovered for any $k<n$; so $c$ is a partial recursive function whose domain is contained in $\{(n, j): j \leq F(n)\}$. For a fixed $i, \alpha$ defines a real $\alpha_{i}$ by $\alpha_{i}(n)=\alpha(i, n)$. Let $\left.V_{n}=\left\{x: \exists j \leq F(n) x \uparrow n=\alpha_{c(n, j)} \uparrow n\right)\right\}$. Since $V_{n}$ is the union of at most $F(n)$ many cones $[x\lceil n]$,

$$
\mathrm{wt}_{\gamma}\left(V_{n}\right) \leq \sum_{j=1}^{F(n)} 2^{-n \gamma}=F(n) 2^{-n \gamma}
$$


which goes effectively to zero by assumption. Thus there is a computable sequence $\left\{n_{k}\right\}_{k \in \mathbb{N}}$ such that $\operatorname{wt}_{\gamma}\left(V_{n_{k}}\right) \leq 2^{-k}$. Let $U_{k}=V_{n_{k}}$. Then $U_{k}$ is $\Sigma_{1}^{0}$ uniformly in $k$, and $x \in \cap_{k} U_{k}$. Hence $x$ is not $\gamma$-random.

Corollary 2 ([2]). No member of a ML-random closed set under the BBCDW distribution is $f$-c.e. for any polynomial-bounded $f$.

Proof. If $f$ is polynomially bounded then clearly $\sum_{j<n} f(i) 2^{-n \gamma}$ goes effectively to zero. Therefore if $x$ is $f$-c.e., $x$ is not $\gamma$-random, hence not a MEMBER $\gamma$ for any $0<\gamma<1$, and thus not a member of a ML-random closed set under the BBCDW distribution.

Computing Brownian slow points. A function $f: \omega \rightarrow \omega$ is diagonally nonrecursive (DNR) if for each $n, f(n)$ is not equal to $\varphi_{n}(n)$, the value (if any) of the $n$th partial recursive function on input $n$. A real $A$ is Kurtz random relative to an oracle $B$ if it does not belong to any $\Pi_{1}^{0}(B)$ subset of $2^{\omega}$ of fair-coin measure zero. Furthermore, $B$ is $\operatorname{Low}(\mathrm{ML}, \mathrm{Kurtz})$ if each real $A$ that is ML-random is Kurtz random relative to $B$.

A starting point for the present paper was the observation $(*)$ that each nonDNR Turing degree is Low(ML, Kurtz). A proof of this result due and credited to Kjos-Hanssen is given by Greenberg and Miller 4]; they prove that the converse holds as well. This can be used to show that each slow point (see Mörters and Peres [12]) of any ML-random Brownian motion must be of DNR Turing degree. The fast points on the other hand form a dense $G_{\delta}$ set, so there are fast points that are 1-generic and hence do not Turing compute any slow points.

In any case, the idea was initially to use the result $\left(^{*}\right)$ to understand members of random closed sets. However, as it turned out one could use the work of Hawkes [5] and Lyons 9 to better effect, in the present paper and in the precursor 6].

\section{Acknowledgments}

The authors thank the Institute of Mathematical Science at Nanjing University (and in particular Liang Yu), where the research leading to Section 2 was carried out in May 2008, for their hospitality. Section 3 contains some earlier results of the second author, who was partially supported by NSF grant DMS-0652669. 


\section{Bibliography}

[1] Logan Axon, Random closed sets and probability, doctoral dissertation, University of Notre Dame, 2009.

[2] George Barmpalias, Paul Brodhead, Douglas Cenzer, Ali Seyyed Dashti, and Rebecca Weber, Algorithmic randomness of closed sets, J. Logic Comput. 17 (2007), no. 6, 1041-1062. MR 2376074

[3] Kenneth Falconer, Fractal geometry, John Wiley \& Sons Ltd., Chichester, 1990. Mathematical foundations and applications. MR 1102677 (92j:28008)

[4] Noam Greenberg and Joseph S. Miller, Lowness for Kurtz randomness, Journal of Symbolic Logic 74 (2009), no. 2, 665-678.

[5] John Hawkes, Trees generated by a simple branching process, J. London Math. Soc. (2) 24 (1981), no. 2, 373-384. MR 631950 (83b:60072)

[6] Bjørn Kjos-Hanssen, Infinite subsets of random sets of integers, Mathematical Research Letters 16 (2009), 103-110.

[7] Bjørn Kjos-Hanssen and Anil Nerode, Effective dimension of points visited by Brownian motion, Theoretical Computer Science 410 (2009), no. 4-5, 347-354.

[8] Jack H. Lutz, Gales and the constructive dimension of individual sequences, Automata, languages and programming (Geneva, 2000), 2000, pp. 902-913.

[9] Russell Lyons, Random walks and percolation on trees, Annals of Probability 18 (1990), no. 3, 931-958. MR 1062053 (91i:60179)

[10] Random walks, capacity and percolation on trees, Annals of Probability 20 (1992), no. 4, 2043-2088. MR 1188053 (93k:60175)

[11] Pertti Mattila, Geometry of sets and measures in Euclidean spaces, Cambridge Studies in Advanced Mathematics, vol. 44, Cambridge University Press, Cambridge, 1995. Fractals and rectifiability. MR 1333890 (96h:28006)

[12] Peter Mörters and Yuval Peres, Brownian Motion. Draft available at http://www.stat.berkeley.edu/ peres/.

[13] Jan Reimann, Effectively closed classes of measures and randomness, Annals of Pure and Applied Logic 156 (2008), no. 1, 170-182.

[14] Jan Reimann and Frank Stephan, Effective Hausdorff dimension, Logic Colloquium '01, 2005, pp. 369-385. 